\title{
Nucleoids Dynamic in Escherichia coli: A Growth Phase Dependent Process
}

\author{
Ali Azam Talukder ${ }^{1 *}$, M Anwar Hossain², Mamoru Yamada ${ }^{3}$ and Akira Ishihama ${ }^{4}$ \\ ${ }^{1}$ Department of Microbiology, Jahangirnagar University, Savar, Dhaka 1342, Bangladesh, ${ }^{2}$ Department of Microbiology, University of Dhaka, \\ Dhaka 1000, Bangladesh, ${ }^{3}$ Department of Biological Chemistry, Faculty of Agriculture, Yamaguchi University, Yamaguchi 753-8515, Japan, \\ ${ }^{4}$ National Institute of Genetics, Mishima, Shizuoka 411-8540, Japan
}

[Received 04 October 2006; Accepted 12 November 2006]

\begin{abstract}
Bacterial DNA compacts in nucleoid bodies. The organization of nucleoid body depends on the association of genomic DNA with a numbers of histone-like proteins. The relax nucleoids organization in rapidly growing Escherichia coli cells associate with six major proteins, Fis, HU, Hfq, H-NS, StpA and IHF, but at stationary phase the nucleoids further tightly pack with Dps. The final steps of compact nucleoids formation occurs with association of MukBEF complex - a bacterial condensin. The change of nucleoid proteins composition in stationary phase accompanies compact DNA organization and genes silencing. Thus, compact nucleoid organization and gene silencing may be crucial for cell survival in stationary phase.
\end{abstract}

Keywords: Escherichia coli, Nucleoid body, Nucleoid proteins, Nucleoid compaction, Condensin

\section{Introduction}

In the nucleus, the genomic DNA of eukaryotic cells organizes into nucleosomes as compact molecules in association with histone proteins. In bacteria such organized nucleosome structure is absent, instead, the bacterial DNA organizes into nucleoid body in association with a sets of specific proteins ${ }^{1-3}$. The $4.7 \mathrm{Mbp}$ DNA (1.5 mm long) of Escherichia coli packs in a highly ordered nucleoid sturcture of $1 \mathrm{~mm}$ long in association with 10-20 DNA binding proteins ${ }^{4-10}$. The most abundant proteins participte in nucleoid formation are Dps (NA-binding protein from starved cells), Fis (f́factor for inversion stimulation), Hfq (host factor for phage $\underline{Q}_{\beta}$ replication), H-NS (histone-like nucleoid structuring protein), HU (heat-unstable nucleoid protein), IHF (integration host factor), MukB (partitioning of sister chromosome), and StpA (suppression of $\underline{t} d^{-}$phenotype $\left.\mathrm{A}\right)^{11-37}$. However, at present the knowledge on the molecular organization of $E$. coli nucleoid is inadequate, because of insufficient evidence on the molecular structure and composition, and its growth phase dependent variation. This review summarizes the growth phase-dependent variations in the structure and protein composition of E. coli nucleoid. Fluctuating patterns of nucleoid proteins and a possible compaction process of genomic DNA into the nucleoids are discussed.

\section{Nucleoid Proteins in E. coli Cells}

In dynamic transition of relax to compact nucleoid formation during the change of growth phase from exponential to stationary depends on sequential participation of a numbers of nucleic acid binding proteins. Some of these directly bind with DNA engaging them in structural change in nucleoid formation, and the other group alters the transcription or translation, thus bringing the morphological change in E. coli ${ }^{38}$. The major DNA binding proteins those involve in growth phase dependent nucleoid organization and gene expression in E. coli are discussed.

Dps

Dps (DNA-binding protein from starved cells) is a starvation or oxidative stress inducible $19 \mathrm{kDa}$ DNA-binding protein in E. coli ${ }^{11}$ and forms a dodecameric complex ${ }^{39}$. Dps is an abundant nucleoid protein in E. coli (Table 1). The purified Dps binds DNA without sequence specificity ${ }^{14}$ and classifies under bacterial nucleoidassociated protein family including HU, H-NS, IHF and Fis 7,16,39. The nucleoid DNA turns into a more compact configuration after binding with Dps ${ }^{16,39-40}$. In exponential phase, Dps exists about 6,000 molecules per cell and gradually increases up to about 180,000 molecules per cell at late stationary phase $\mathrm{p}^{16,32}$.

Fis

Fis (factor for inversion stimulation) is a small basic DNA-binding protein in E. coli originally identified as a factor for site-specific DNA recombination ${ }^{41}$ and participates in transcription of the growth-related genes and DNA replication ${ }^{42}$. Fis protein level depends on growth phase of $E$. coli, and in exponential phase it estimates $\sim 60,000$ molecules per cell, but becomes undetectable in the stationary phase (Table 1). The synthesis of Fis stops at stationary phase, resulting decrease of intracellular level by 500 to 1,000 fold $^{16-17}$.

*Corresponding author:

Dr. Ali Azam Talukder, Assistant Professor, Department of Microbiology, Jahangirnagar University, Saver, Dhaka 1342, Bangladesh Tel (Office): (02) 7708478-85/1566; Fax: +880 (02) 7708069.E-mail: azam115@hotmail.com 
Table 1. General properties of the nucleoid proteins in Escherichia coli

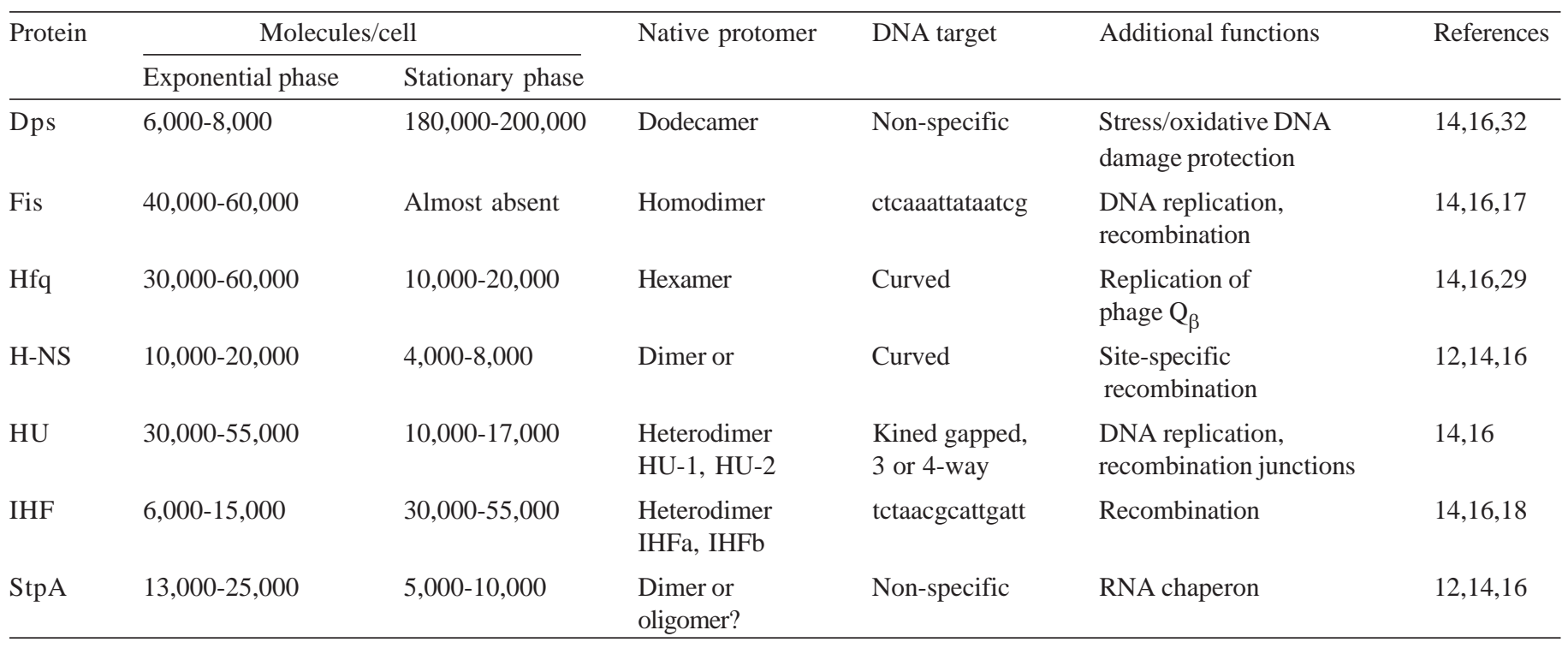

\section{Hfq}

Hfq (host factor for phage $\mathrm{Q}_{\beta}$ replication, HF-I), a nucleic acidbinding protein, recognizes as host factor involving in replication of phage $\mathrm{Q}_{\beta} \mathrm{RNA}^{28-29}$. Hfq preferentially binds to curved-DNA sequence in a non-specific manner ${ }^{14}$. Hfq found $~ 55,000$ molecules per cell in exponential phase (Table 1) and assembles in nucleoid body and ribosomes ${ }^{16,28}$. Hfq controls the translation of the $\sigma$ factor gene rpoS and the DNA repair gene $m u t S^{43-44}$.

\section{$H-N S$}

H-NS (histone-like nucleoid structuring protein) is a wellcharacterized nucleoid-associated protein repressing global transcription that affects more than 100 genes or operons in E. coli ${ }^{12}$. The number of H-NS molecules reaches $\sim 20,000$ per cell in exponential phase, but decrease to $40 \%$ at late stationary phase $^{16}$. Remarkably, the pattern of $\mathrm{H}-\mathrm{NS}$ growth-dependent variation is similar to those of Hfq, HU and StpA (Table 1).

\section{$H U$}

HU (heat-unstable nucleoid protein) is considered as a prokaryotic homologue of eukaryotic histones ${ }^{19}$, but the sequence comparison indicates that $\mathrm{HU}$ is more analogous to the eukaryotic high morbility group (HMG) proteins ${ }^{45}$. HU exists in solution as a heterodimer consisting of two similar subunits. Like Hfq, HU is also associated with ribosomes ${ }^{46}$. About 30,000 to 55,000 HU molecules may exist in the exponentially growing cell of E. coli W3110. HU dimers may associate every 300-400 bp of the E. coli genome under the saturation condition. Upon entry into stationary phase, the HU level gradually decreases to less than one third of the maximum level in late stationary phase ${ }^{16}$.

\section{IHF}

IHF (integration host factor), the most abundant sequence-specific DNA-binding proteins in E. coli, involves in the integration of phage $\lambda$ DNA into host DNA ${ }^{14,47-48}$. Currently, IHF recognizes as a global transcription regulatory factor for many genes ${ }^{49}$. IHF may exist 12,000 molecules per cell in exponential phase and the number increases about 5-fold in early stationary phase ${ }^{16,18}$. At the transient state, IHF becomes the second most abundant protein, suggesting IHF plays crucial roles in transitional conversion of nucleoids structure from more relax to compact form during the phase transition of cell growth.

MukB

MukB (partitioning of sister chromosome), a 177-kDa protein, involves in partition of the bacterial chromosome. An estimated $\sim 150$ molecules of MukB are present per cell ${ }^{30}$. The $m u k B$ mutation causes unfolding of the nucleoid ${ }^{50}$. In addition, the domain structure of MukB resembles eukaryotic and bacterial structural maintenance of chromosome (SMC) proteins that interact with topoisomerases in fission yeast and Drosophila ${ }^{51}$.

\section{StpA}

StpA (suppression of $t d^{-}$phenotype A) is a multi-copy suppressor of a $t d^{-}$phenotype of phage $\mathrm{T}^{52}$. The growing $E$. coli W3110 cells contain $\sim 25,000$ molecules of StpA whereas in stationary phase have $\sim 7,000-8,000$ molecules $^{16}$. The sequence identity (58\%) and growth-dependent variation of StpA and H-NS suggests that these two proteins perform overlapping functions. Indeed, both proteins have similar sequence recognition specificity and DNA-binding affinity ${ }^{14}$.

\section{Compact and Stress Resistant Stucture Formed by E. coli at Stationary Growth Phase}

At stationary phase, E. coli alters the structures of cell wall, cytoplasm and nucleoid as well as the number of cellular components (Figure 1) and cells become highly resistant to a variety of stresses. The cell volume, and the chemical composition and structure of cell envelope containing the outer membrane, cell wall, and cytoplasmic or inner membrane drastically changes when $E$. coli enter in stationary phase. There is an increase in 
cardiolipin and cyclopropyl fatty acid derivatives at the inner membrane (Figure 1) ) $^{53-54}$. Stationary phase cells also have a higher protein/lipid ratio in the membranes, which makes them less prone to lateral phase separation and a higher degree of crosslinking among membrane proteins ${ }^{55-56}$. The cell wall become thick in stationary phase containing 4-5 layers of peptidoglycan compared to 2 or 3 layers in exponential growth, contributing stability of the envelopes of stationary-phase cells ${ }^{56}$.

The transcription of each set of genes under specific growthcondition by DNA-dependent RNA polymerase depends on the presence of one of the seven $\sigma$ subunits plus core enzyme. The synthesis of $\sigma^{\mathrm{S}}$ is regulated by ppGpp and H-NS ${ }^{57-58}$. The level of $\sigma^{\mathrm{S}}$ increases and selective activation of $\mathrm{E} \sigma^{\mathrm{S}}$ holoenzyme may occur due to accumulation of trehalose in stationary growth phase ${ }^{59-60}$. Decreasing linking number of supercoiled DNA in stationary phase enhances the activity of $\sigma^{\mathrm{S}}$ holoenzyme ${ }^{61-62}$.

Translation machinery adapts in stationary phase by forming translationally incompetent $100 \mathrm{~S}$ ribosome, in which $\mathrm{Rmf}$ (ribosome modulating factor) is involved. When stationary phase culture transfer into fresh medium, the ribosome dimers, 100S, are converted into $70 \mathrm{~S}$ monomers within a few minutes ${ }^{63}$. E. coli $\mathrm{rmf}^{-}$ mutant cannot survive in stationary phase, implicating ribosome dimerization is presumably essential for stationary phase survival $^{64-65}$.

\section{Mechanisms of Nucleiod Body Formation in E. coli}

Bacterial nucleiod is continously remodleed by the defined sets of genes expression to fit the needs of the physiological state of growth of it. In E. coli nucleoid body formation and compaction, is growth phase dependent process, and the most striking changes occur during the phase switching from exponential to stationary phase (Figure 2). Similar compaction of DNA is observed in other microorganisms Listeria monocytogenes and Lactobacillus plantarum ${ }^{66-67}$. In exponential phase E. coli DNA form less compact supercoiled nucleoid than those in the stationary phase, possibly due to presence of molar excess of Fis over Dps (Figure 1 and 2).

\section{Nucleoid Compaction}

In E. coli, several factors involve in the compaction of chromosomal DNA into the nucleoid. The size of compacted nucleoid was estimated by measurement of the areas of the fluorescence images of individual nucleoids, with a user-independent thresholding procedure $^{68}$. The average thresholded area of compacted nucleoids is $1.4 \mu \mathrm{m}^{2}$. Whereas the average area for the expanded nucleoids is $2.8 \mu \mathrm{m}^{2}$ as released by the low salt-spermidine procedure ${ }^{38,69}$. Nucleoids in rapidly growing cells are complex structures with multiple genome equivalents of DNA at different stages of replication associated with large amounts of proteins and other ligands ${ }^{16,69-72}$. Model studies indicate that changes in these levels of compaction can have important effects upon the ability of the DNA to function in a wide variety of enzymatic and chemical reactions ${ }^{68}$. Many reactions related to DNA are accelerated by compaction ${ }^{68,73-75}$, but the rate of transcription decreases ${ }^{59}$. The current review is part of an attempt to better understand the factors that control DNA compaction. A variety of DNA-compacting factors have been suggested $^{3,19,68,71,76-78}$.

\section{E. coli (exponetial phase)}

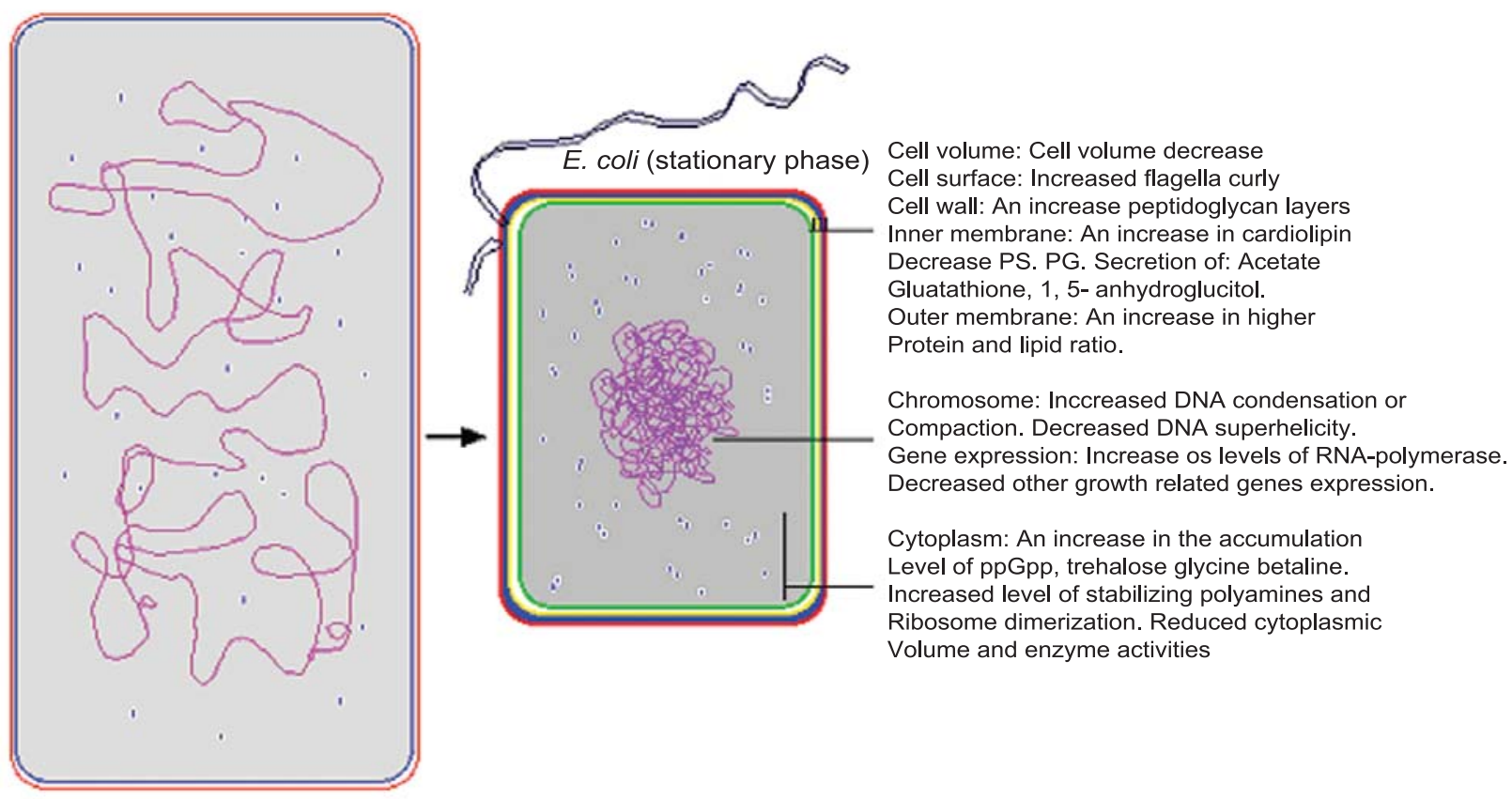

Figure 1. Overall changes in Escherichia coli cell entering into stationary growth phage. 


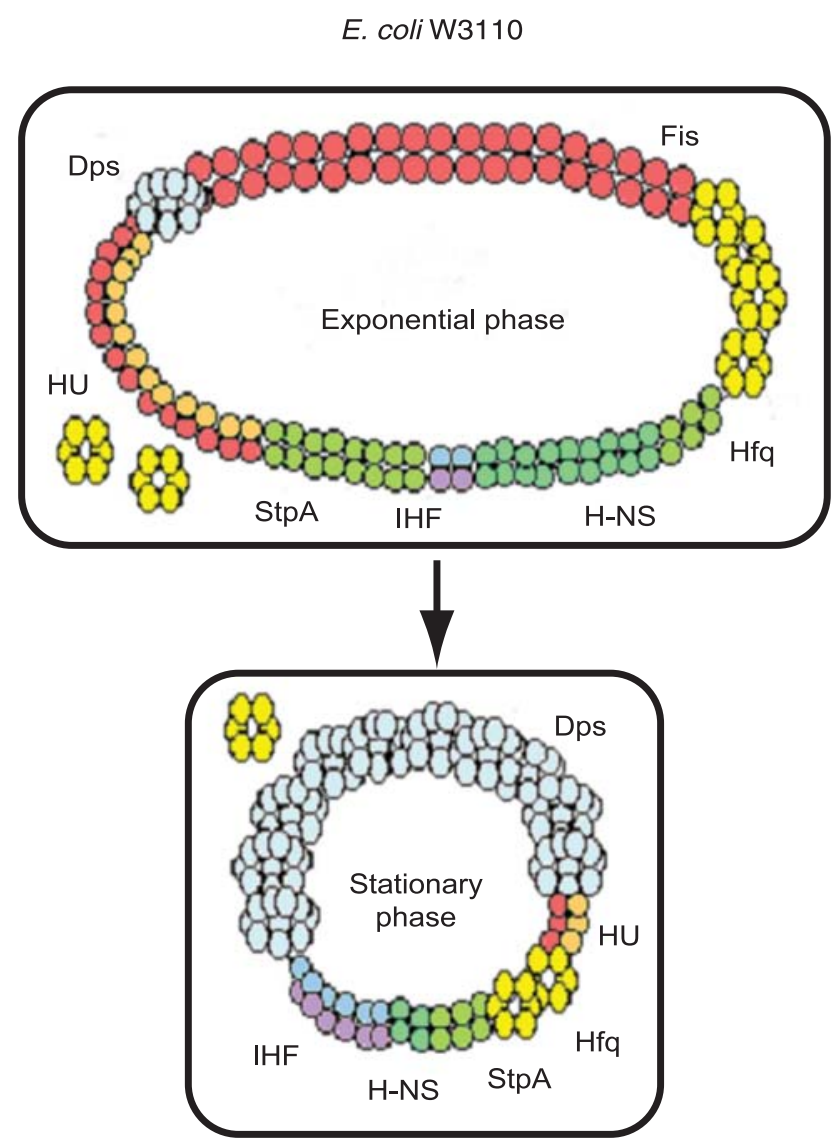

Figure 2. Schematic representation of protein composition ans structure of Escherichia coli nucleoids and their variations in two different growth phases. The intracellular concentrations of nucleoid proteins in E. coli were converted into the number of molecules per cell. All the nucleoid proteins are assumed to be associated with the nucleoid. The nucleoid structure in stationary phase is more tightly compacted than that in exponential phase. The oeder of accumulation level in exponentially growing E. coli is: Fis (27\%) $>\mathrm{Hfq}(24 \%)$ $>H U(23 \%)>\operatorname{StpA}(10 \%)>H-N S(7 \%)>I H F(6 \%)>D p s(3 \%)$. The major protein components of nucleoid change from Fis, $\mathrm{Hfq}$ and $H U$ in the exponential phase to Dps in the stationary phase. The order of abundance in the stationary shase is: Dps (70\%) $>\operatorname{IHF}(15 \%)>H U(5 \%)>H f q(5 \%)>S t p A(3 \%)>H-N S(2 \%)$ $>$ Fis $(0 \%)$.

\section{Growth phase}

Stationary phase nucleoids are much more compacted compared to exponential phase nucleoids in intact cells ${ }^{16}$. Sedimentation profiles ${ }^{38}$ and atomic force microscopy ${ }^{40}$ appearence studies of E. coli suggests that nucleoids in stationary phase is condensed more than that of exponential phase. Therefore, chromosome compaction/ condensation occurs at the transition step from exponential phase to stationary phase where DNA superhelicity decreases (Figure 3).

\section{Supercoiling}

DNA is negatively supercoiled in vivo ${ }^{42,78-80}$. Half of the supercoiling controls a number of nucleoid-associated proteins,
DNA

Nucleoid proteins

Fundamenta structure of nucleoid in phase

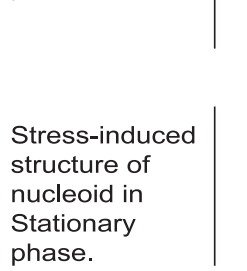

MukBEF complexes

\section{Final round} of DNA compaction by MukBEF complexes Exponential
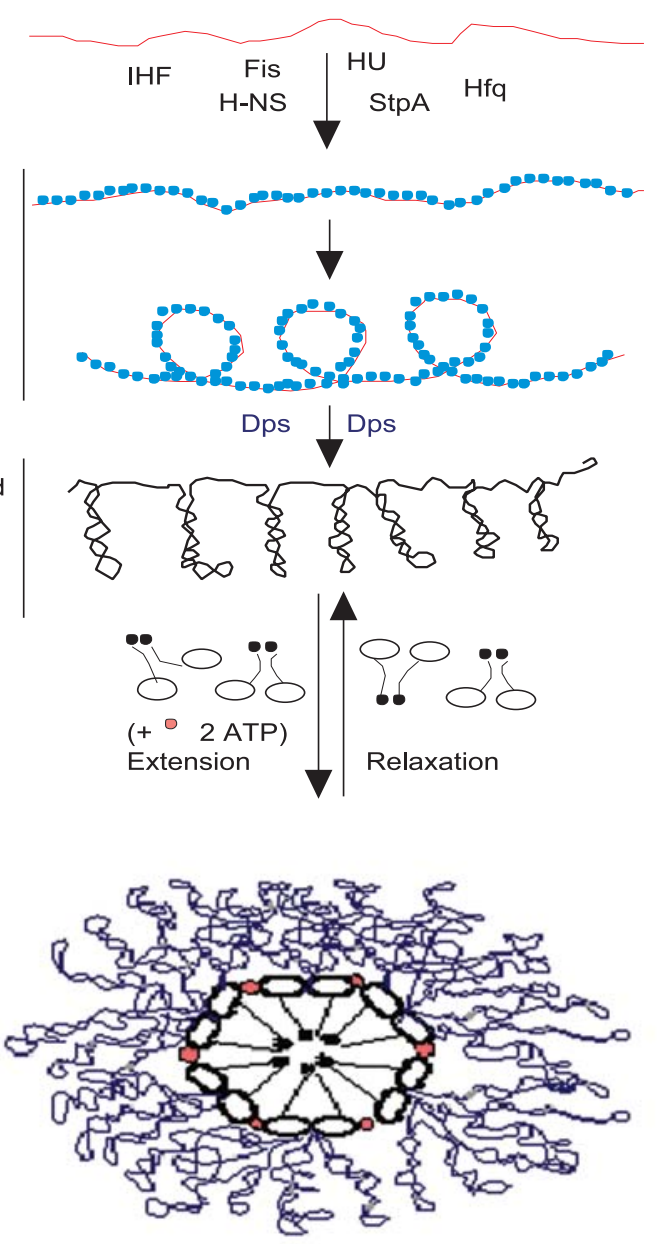

Figure 3. A model for the DNA compaction mechanism by histone-like proteins (Fis, Hfq, H-NS, HU, IHF, StpA and Dps) and bacterial condensing, MukBEF. The DNA and histone-like proteins form a bacterial chromatin-like structure, nucleoid. The nucleoid, which becomes tightly compacted and forms a coral reef structure with several supercoiled loops in stationary phase, is mainly mediated via Dps. The final round of DNA condensation is mediated via MukBEF complexes (with a central hinge, DNA and two ATPs between adjacent heads are shown), in which 30-kb loops of supercoiled DNA form topological domains between heads of individual helically propagated MukBEF complexes. The bottom part of this figure is adapted from Case et al. ${ }^{93}$.

which have been implicated in the organization of bacterial nucleoid, with additional roles in transcription, recombination, and replication ${ }^{59}$. The remaining half of its supercoiling is introduced into DNA molecules by enzymes called DNA topoisomerases ${ }^{80-82}$. In E. coli, the level of supercoiling depends mainly on the activities of DNA TopI (topoisomerase I) and TopII (gyrase) and, to a lesser extent, on that of TopIV ${ }^{80-81,83}$. TopI introduces single strand breaks into DNA molecules and causes their relaxation. Gyrase, an ATPdependent enzyme composed of two GyrA and two GyrB subunits, makes double-strand breaks and introduces supercoils into DNA molecules. TopIV is an ATP-dependent enzyme that makes double- 
strand breaks and contributes to DNA relaxation ${ }^{82-83}$. The level of DNA supercoiling may be regulated by a complex homeostatic control ${ }^{83-84}$. Transcription of topA, encoding TopI, increases when the supercoiling level is high, whereas transcription of gyrA and gyrB, encoding GyrA and GyrB, respectively, increases when the supercoiling level is low ${ }^{84}$. It is well established that the supercoiling level influences the activity of many promoters ${ }^{82}$ and that environmental stresses alter this level ${ }^{61-62,85}$.

The linking number of supercoiled DNA decreases in stationary phase under different physiological conditions including high temperature, oxidative stress, $\mathrm{NaCl}$ and extreme $\mathrm{pH}^{61,86-87}$. Stationary phase cells may have relaxed plasmids, which are converted to supercoiled structures by gyrase as soon as nutrients become available ${ }^{86,88}$. Stationary phase rpoS mutant cells show a bimodal distribution of plasmids and fail to supercoil plasmids after the addition of nutrients, suggesting that rpoS plays a role in the regulation of plasmid topology during stationary phase $^{88}$. Dps is also involved in the negative supercoiling of the E. coli genomic DNA ${ }^{38}$. Both the RpoS and Dps levels are more than 10 -times higher in stationary phase than in exponential phase as mentioned earlier ${ }^{16,38}$.

\section{Dps}

The tight compaction of nucleoid in stationary phase is mediated by $\mathrm{Dps}^{40}$. However, Dps expression in exponential phase is unable to induce the nucleoid compaction, presumably due to the presence of some proteins, which prevent Dps from binding to DNA. Overexpression of Dps induced an intracellular crystalline structure in vivo ${ }^{89-90}$, and purified Dps proteins were co-crystallized with DNA $^{89-92}$. The highly compacted nucleoids observed in stationary phase appear to have similar characteristics to a biocrystal that is resistant to the detergent treatment ${ }^{40}$. Bacterial cells might protect their own nucleoids from environmental stresses including chemicals by tight compaction. Interestingly, the $d p s$ mutant cells are very sensitive to environmental stresses ${ }^{11,31}$. The Dps protein is thus crucial for achieving higher ordered structures ${ }^{16,40,89-91}$, and for protecting the stresses.

Formation of the coral reef structure might be the first step towards the tight compaction as suggested by Kellenberger and his co-worker ${ }^{92}$. Recent finding suggested that the bacterial condensin, MukBEF complex is involved in compaction of the genome DNA ${ }^{94}$. Condensins are conserved proteins containing SMC (structural maintenance of chromosomes) moieties. It is well characterized that eukaryotic condensins stabilize DNA supercoil in vitro ${ }^{95,97}$, and prokaryotic condensins affect the supercoiling state of the nucleoid in vivo ${ }^{72,98}$. MukBEF contains an ATP-binding domain in the N-terminal region and a DNA-binding domain in the C-terminal region ${ }^{99}$. Mutational inactivation of any of the three $m u k$ genes results temperature-sensitive growth, and expanded and disordered nucleoids containing cells ${ }^{100}$. A mutation in the TopI gene suppresses the Muk phenotype, probably because the absence of TopI leads to an increased compaction of the nucleoid by the additional negative supercoils ${ }^{101-102}$. The cellular concentration of MukBEF in vivo is about one MukBEF per $\sim 30 \mathrm{~kb}$ of $E$. coli genome DNA ${ }^{30}$. Because the nucleoid is already condensed by DNA binding proteins and negative supercoiling $3,8,16,59$, MukBEF-binding sites that are $30 \mathrm{~kb}$ apart on the genome may be trapped in close proximity between adjacent MukBEF heads. Moreover, MukBEF localizes to the center of the nucleoid ${ }^{34}$. Therefore, MukBEF would also be an important contributor to the final round of compaction of the genomic DNA into the folded chromosome with several topological domains (Figure 3) )1,94,102. $^{5}$.

\section{Conclusion}

We propose two models for growth phase-dependent changes in the structure and protein composition of $E$. coli nucleoids (Figures 2 and 3). Firstly, relaxed form of nucleoids in rapidly growing cells are mainly organized by 6 major proteins, Fis, HU, Hfq, H-NS, StpA and IHF. Secondly, ins stationary phase, the concomitant decreased of FIS and increase of polyamine might be involved in forming compacted nucleoid structure from relaxed DNA. Increase of Dps in molar excess to Fis might re-fold and supercoil the DNA negatively. Both of these process occur simultenously. Then, the negative super-coiled structured DNA folded back and Dps further deposited tightly on the folded back structure. Thus, a highly compact nucleoid form of coral reef structures with several supercoiled domains in the stationary phase of growth is formed. The final step of nucleoid compaction occurs in the presence of MukBEF complex which binds and induces further condensation of dsDNA, thereby creating topologically isolated domains (Figure 3). This remodelling of nucleiod regulates the expression of defined sets of genes to fit the needs of cells under specific growth condition.

\section{References}

1. Hecht RM, Stimpson D \& Pettijohn DE. 1977. Sedimentation properties of the bacterial chromosomes as an isolated nucleoid and as an unfolded DNA fiber: Chromosomal DNA folding measured by rotor speed effects. $J$ Mol Biol. 111: 257-277.

2. Hecht RM, Taggart RT \& Pettijohn DE. 1975. Size and DNA content of purified $E$. coli nucleoids observed by fluorescence microscopy. Nature. 253: 60-62.

3. Pettijohn DE. 1996. The nucleoid. In Escherichia coli and Salmonella (Neidhardt FC, Ingraham JL, Low KB, Magasanik, Schaechter \& Umbarger ME eds), pp 158-166. American Society for Microbiology, Washington DC.

4. Helmann JD \& Chamberlin MJ. 1988. Structure and function of bacterial sigma factors. Annu Rev Biochem. 57: 839-872.

5. Hinnebusch B \& Bendich AJ. 1997. The bacterial nucleoid visualized by fluorescence microscopy of cells lysed within agarose: Comparison of Escherichia coli and Spirochetes of the genus Borrelia. J Bacteriol. 179: 2228-2237.

6. Pettijohn DE. 1982. Structure and properties of the bacterial nucleoid. Cell. 30: 667-669. 
7. Schmid MB. 1990. More than just 'histone-like' proteins. Cell. 63: 451-453.

8. Trun N \& Marko JF. 1998. Architecture of a bacterial chromosome. ASM News. 64: 276-283.

9. Woldringh CL \& Nanninga N. 1985. Structure of the nucleoid and cytoplasm in the intact cell. In Molecular Cytology of Escherichia coli (Nenninga N ed), pp 161-197, Academic Press, London.

10. Worcel A \& Burgi E. 1972. On the structure of the folded chromosome of Escherichia coli. J Mol Biol. 71: 127-147.

11. Almirón M, Link AJ, Furlong D \& Kolter R. 1992. A novel DNA binding protein with regulatory and protective roles in starved Escherichia coli. Genes Dev. 6: 2646-2654.

12. Atlung T \& Ingmer H. 1997. H-NS: A modulator of environmentally regulated gene expression. Mol Microbiol. 24: 7-17.

13. Azam TA, Hiraga S \& Ishihama A. 2000. Two types of localization of the DNA-binding proteins within the Escherichia coli nucleoid. Genes Cells. 5: 613-626.

14. Azam TA \& Ishihama A. 1999. Twelve species of the nucleoid-protein from Escherichia coli: Sequence recognition specificity and DNAbinding affinity. J Biol Chem. 274: 33105-33113.

15. Azam TA \& Ishihama A. Growth phase-dependent changes in the structure and composition of the Escherichia coli nucleoid (Unpublished data).

16. Azam TA, Iwata A, Nishimura A, Ueda S \& Ishihama A. 1999. Growth phase-dependent variation in protein composition of the Escherichia coli nucleoid. J Bacteriol. 181: 6361-6370.

17. Ball CA, Osuna R, Ferguson KC \& Johnson RC. 1992. Dramatic changes in Fis levels upon nutrient upshift in Escherichia coli. J Bacteriol. 174: 8043-8056.

18. Ditto MD, Roberts D \& Weisberg RA. 1994. Growth phase variation of Integration Host Factor level in Escherichia coli. J Bacteriol. 176: 3738-3748.

19. Drlica K \& Rouviere-Yaniv J. 1987. Histone-like proteins of bacteria. Microbiol Rev. 51: 301-319.

20. Dürrenberger M, Teana AL, Citro G, Venanzi F, Gualerzi CO \& Pon CL. 1991. Escherichia coli DNA-binding protein H-NS is located in the nucleoid. Res Microbiol. 142: 373-380.

21. Dürrenberger M, Bjornsti MA, Uetz T, Hobot JA \& Kellenberger E. 1988. Intracellular localization of the histone like protein $\mathrm{HU}$ in Escherichia coli. J Bacteriol. 170: 4757-4768.

22. Jishage M \& Ishihama A. 1995. Regulation of RNA polymerase sigma subunit synthesis in Escherichia coli: Intracellular levels of $\sigma^{70}$ and $\sigma^{38}$. J Bacteriol. 177: 6832-6835.

23. Jishage M, \& Ishihama A. 1997. Variation in RNA polymerase sigma subunit composition within different stocks of Escherichia coli W3110. J Bacteriol. 179: 959-963.

24. Jishage M \& Ishihama A. 1998. A stationary phase protein in Escherichia coli with binding activity to the major $\sigma$ subunit of RNA polymerase. Proc Natl Acad Sci USA. 95: 4953-4958.

25. Jishage M, Iwata A, Ueda S \& Ishihama A. 1996. Regulation of RNA polymerase sigma subunit synthesis in Escherichia coli: Intracellular levels of four species of sigma subunit under various growth conditions. J Bacteriol. 178: 5447-5451.

26. Jiang W, Hou Y \& Inoue M. 1997. CspA, the major cold-shock protein of Escherichia coli is an RNA chaperon. J Biol Chem. 272: 196-202.

27. Jones PG \& Inoue M. 1994. The cold-shock response - A hot topic. Mol Microbiol. 11: 811-818.

28. Kajitani M, Kato A, Wada A, Inokuchi Y \& Ishihama A. 1994 Regulation of the Escherichia coli hfq gene encoding the host factor for phage Qb. J Bacteriol. 176: 531-534.
29. Kajitani M \& Ishihama A. 1991. Identification and sequence determination of the host factor gene for bacteriophage $\mathrm{Q}_{\beta}$. Nucleic Acids Res. 19: 1063-1066.

30. Niki H, Jaffe A, Imamura R, Ogura T \& Hiraga S. 1991. The new gene mukB codes for a $177 \mathrm{kd}$ protein with coiled-coil domains involved in chromosome partitioning of E. coli. EMBO J. 10: 183-193.

31. Ishihama A. 1999. Modulation of the nucleoid, the transcription apparatus, and the translation machinery in bacteria for stationary phase survival. Genes Cells. 3: 135-143.

32. Martinez A \& Kolter R. 1997. Protection of DNA during oxidative stress by the nonspecific DNA-binding protein Dps. J Bacteriol. 179: 5188-5194.

33. Morikawa K, Ohniwa RL, Kim J, Murayama A, Ohta T \& Takeyasu K. 2006. Bacterial nucleoid dynamics: Oxidative stress response in Staphylococcus aureus. Genes cells. 11: 49-423.

34. Ohsumi K, Yamazoe M \& Hiraga S. 2001. Different localization of seqA-bound nascent DNA clusters and MukF-MukE-MukB complex in Escherichia coli cells. Mol Microbiol. 40: 835-845.

35. Rimisky S. 2004. Structure of the histone-like protein H-NS and its role in regulation and genome superstructure. Cur Opinion Microbiol. 7: 109-114.

36. Rouviere-Yaniv J, Yaniv M \& Germond JE. 1979. Escherichia coli DNA-binding protein HU forms nucleosome-like structure with circular double-stranded DNA. Cell. 17: 265-274.

37. Slater S, Wold S, Lu M, Boye E, Skarstad K \& Kleckner N. 1995. E. coli SeqA protein binds oriC in two different methyl-modulated reactions appropriate to its roles in DNA replication initiation and origin sequestration. Cell. 82: 927-936.

38. Azam TA. 2005. Survival and death in bacteria. In Structure and Composition of Escherichia coli Nucleoids (Yamada M ed), Vol. 2, pp 77-101. Research Signpost, Kerala.

39. Grant RA, Filman DJ, Finkel SE, Kolter R \& Hogle JM. 1998. The crystal structure of Dps, a ferritin homolog that binds and protects DNA. Nat Stru Biol. 5: 294-303.

40. Kim J, Yoshimura SH, Hizume K, Ohniwa RL, Ishihama A \& Takeyasu K. 2004. Fundamental structural units of the Escherichia coli nucleoid revealed by atomic force microscopy. Nucleic Acids Res. 32: 19821992.

41. Finkel SE \& Johnson RC. 1992. The Fis protein: It's not just for DNA inversion anymore. Mol Microbiol. 6: 3257-3265.

42. Thompson JF, Moitoso de Vargas L, Koch C, Kahmann R \& Landy A. 1987. Cellular factors couple recombination with growth phase: characterization of a new component in the lambda site-specific recombination pathways. Cell. 50: 901-908.

43. Schuppli D, Miranda G, Tsui HC, Winkler ME, Sogo JM \& Weber H. 1997. Altered 3'-terminal RNA structure in phage $\mathrm{Q}_{\beta}$ adapted to host factor-less Escherichia coli. Proc Natl Acad Sci USA. 94: 1023910242.

44. Muffler A, Fischer D \& Hengge-Aronis R. 1996. The RNA-binding protein HF-I, known as a host factor for phage $\mathrm{Q}_{\beta}$ RNA replication, is essential for rpoS translation in Escherichia coli. Genes Dev. 10: $1143-1151$

45. Oberto J, Drlica K \& Rouviere-Yaniv J. 1994. Characterization of histone-like protein HU in Escherichia coli. Biochimie. 76: 901913.

46. Suryanarayana T \& Subramanian AR. 1978. Specific association of two homologous DNA-binding proteins to the native 30S ribosomal subunits of Escherichia coli. Biochem Biophys Acta. 520: 342-357.

47. Craig NL \& Nash SE. 1984. E. coli integration host factor binds to specific sites in DNA. Cell. 39: 707-716. 
48. Nash HA, \& Robertson CA. 1981. Purification and properties of the Escherichia coli protein factor required for lambda integration recombination. J Biol Chem. 256: 9246-9253.

49. Ishihama A. 1997. Promoter selectivity control of Escherichia coli RNA polymerase. In Nucleic Acids Molecular Biology (F Eckstein and D Lilley eds), Vol 11, Mechanism of Transcription, pp 53-70. Springer-Verlag, Heidelberg.

50. Wang Q, Mordukhoba EA, Edwards AL \& Rybenkov VV. 2006. Chromosome condensation in the absence of the non-SMC subunits of MukBEF. J Bacteriol. 188: 4431-4441.

51. Hirano T. 1999. SMC-mediated chromosome mechanics: A conservative scheme from bacteria to vertebrates? Genes Dev. 13: 11-19.

52. Zhang Z \& Belfort M. 1992. Nucleotide sequence of a newly identified Escherichia coli gene, stpA, encoding an H-NS-like protein. Nucleic Acids Res. 20: 6734.

53. Grogan DW \& Cronan JE. 1997. Cyclopropane ring formation in membrane lipids of bacteria. Microbiol Mol Biol Rev. 61: 429-441.

54. Kusano S \& Ishihama A. 1997. Stimulatory effect of trehalose on the formation and activity of Escherichia coli RNA polymerase $E \sigma^{38}$ holoenzyme. J Bacteriol. 179: 36493654.

55. Kornberg A. 1995. Inorganic polyphosphate: Toward making a forgotten polymer unforgettable. J Bacteriol. 177: 491-496.

56. Mirelman D \& Siegel RC. 1979. Oxidative deamination of aminolysine residues and formation of Schiff base cross linkages in cell envelopes of Escherichia coli. J Biol Chem. 254: 571-574.

57. Lange R, Fischer D \& Hengge-Aronis R. 1995. Identification of the transcriptional start sites and the role of ppGpp in the expression of rpoS, the structural gene for the $\sigma^{\mathrm{S}}$ subunit of RNA polymerase in Escherichia coli. J Bacteriol. 177: 4676-4680.

58. Gentry DR, Hernandez VJ, Nguyen LH, Jensen DB \& Cashel M. 1993. Synthesis of the stationary-phase sigma factor $\sigma^{\mathrm{S}}$ is positively regulated by ppGpp. J Bacteriol. 175: 7982-7989.

59. El-Khani MA \& Stretton RJ. 1981. Effect of growth medium on the lipid composition of log and stationary phase cultures of Salmonella typhimurium. Microbios. 31: 161-170.

60. Bordes P, Conter A, Morales V, Bouvier J, Kolb A \& Gutierrez C. 2003. DNA supercoiling contributes to disconnect $\sigma^{\mathrm{S}}$ accumulation from $\sigma^{\mathrm{S}}$-dependent transcription in Escherichia coli. Mol Microbiol. 43: 561-571.

61. Ishihama A. 1997. Adaptation of gene expression in stationary phase bacteria. Curr Opinion Genet Dev. 7: 582-588.

62. Weitao T, Nordstrom K \& Dasgupta S. 2000. Escherichia coli cell cycle control genes affect chromosome superhelicity. EMBO Rep. 1: 494-499.

63. Yamagishi M, Matsushima H, Wada A, Sakagami M, Fujita N \& Ishihama A. 1993. Regulation of the Escherichia coli rmf gene encoding ribosome modulation factor (RMF): Growth phase- and growth ratedependent control. EMBO J. 12: 625-630.

64. Wada A, Yamazaki Y, Fujita N \& Ishihama A. 1990. Structure and probable genetic location of a ribosome modulation factor associated with 100S ribosomes in stationary-phase Escherichia coli. Proc Natl Acad Sci USA. 87: 2657-2661.

65. Wada A. 1998. Growth phase coupled modulation of Escherichia coli ribosomes. Genes Cells. 3: 203-208.

66. Wouters PC, Glaasker E \& Smelt JPPM. 1998. Effects of high pressure on inactivation kinetics and events related to proton efflux in Lactobacillus plantarum. Appl Environ Microbiol. 64: 509-514.

67. Mackey B, Forestie're K, Isaacs NS, Stenning R \& Brooker B. 1994. The effect of high hydrostatic pressure on Salmonella thompson and
Listeria monocytogenes examined by electron microscopy. Lett Appl Microbiol. 19: 429-432.

68. Zimmerman SB. 2002. Toroidal nucleoids in Escherichia coli exposed to chloramphenicol. J Struct Biol. 138: 199-206.

69. Murphy LD \& Zimmerman SB. 1997. Stabilization of compact spermidine nucleoids from Escherichia coli under crowded conditions: Implication for in vivo nucleoid structure. J Struct Biol. 199: 321335.

70. Flashner Y \& Gralla JD. 1988. DNA dynamic flexibility and protein recognition-differential stimulation by bacterial histone-like protein HU. Cell. 54: 713-721.

71. Murphy LD \& Zimmerman SB. 1997. Isolation and characterization of spermidine nucleoids from Escherichia coli. J Struct Biol. 199: 336-346.

72. Yamashino T, Ueguchi C \& Mizuno T. 1995. Quantitative control of the stationary phase-specific sigma factor, $\sigma^{\mathrm{S}}$ in Escherichia coli: Involvement of the nucleoid protein H-NS. EMBO J. 14: 594-602.

73. Zimmerman SB. 2004. Studies on the compaction of isolated nucleoids from Escherichia coli. J Struct Biol. 147: 146-158.

74. Balke VL \& Gralla JD. 1987. Changes in the linking number of supercoiled DNA accompany growth transitions in Escherichia coli. J Bacteriol. 169: 4499-4506.

75. Pettijohn DE. 1988. Histone-like proteins and bacterial chromosome structure. J Biol Chem. 263: 12793-12796.

76. Zimmerman SB \& Murphy LD. 2001. Release of compact nucleoids with characteristic shapes from Escherichia coli. J Bacteriol. 183: 5041-5049.

77. Reece RJ \& Maxwell A. 1991. DNA gyrase: Structure and function. Crit Rev Biochem Mol Biol. 26: 335-375.

78. Abril AM, Salas M \& Hermoso JM. 2002. The in vivo function of phage 29 nucleoid-associated protein p6 requires formation of dimmers. Gene. 296: 187-194.

79. Champoux JJ. 2001. DNA topoisomerases: Structure, function, and mechanism. Annu Rev Biochem. 70: 369-413.

80. Wang JC. 1996. DNA topoisomerases. Annu Rev Biochem. 65: 635692.

81. Zechiedrich EL, Khodursky AB, Bachellier S, Schneider R, Chen D, Lilley DMJ \& Cozzarelli NR. 2000. Roles of topoisomerases in maintaining steady-state DNA supercoiling in Escherichia coli. J Biol Chem. 275: 8103-8113.

82. Rui S \& Tse-Dinh YC. 2003. Topoisomerase function during bacterial responses to environmental challenge. Front Biosci. 8: 256-263.

83. Menzel R \& Gellert M. 1983. Regulation of the genes for E. coli DNA gyrase: Homeostatic control of DNA supercoiling. Cell. 34: 105-113.

84. Pettijohn DE \& Sinder RR. 1985. Structure of the isolated nucleoid. In Molecular Cytology of Escherichia coli (Nanninga N ed), pp 199227. Academic Press, London.

85. Conter A. 2003. Plasmid DNA supercoiling and survival in long-term cultures of Escherichia coli: Role of NaCl. J Bacteriol. 185: 53245327.

86. Ogata Y, Mizushima T, Kataoka K, Miki T \& Sekimizu K. 1994. Identification of DNA polymerases involved in immediate and transient DNA relaxation induced by heat shock in Escherichia coli. Mol Gen Genet. 244: 451-455.

87. Dominguez YR, Contreras FG, Ramirez-Santos J, MembrilloHernandez J \& Gomez-Eichelmann MG. 2003. Plasmid DNA supercoiling and gyrase activity in Escherichia coli wild-type and rpoS stationary-phase cells. J Bacteriol. 185: 1097-1100. 
88. Wolf SG, Frenkiel-Krispin D, Arad T, Finkel SE, Kolter R \& Minsky A. 1999. DNA protection by stress-induced biocrystalization. Nature. 400: 83-85.

89. Frenkiel-Krispin D, Levin-Zaidman S, Shimoni E, Wolf SG, Wachtel EJ, Arad T, Finkel SE, Kolter R \& Minsky A. 2001. Regulated phase transitions of bacterial chromatin: A non-enzymatic pathway for generic DNA protection. EMBO J. 20: 1184-1191.

90. Frenkiel-Krispin D, Ben-Avraham I, Englander J, Shimoni E, Wolf SG \& Minsky A. 2004. Nucleoid restructuring in stationary-state bacteria. Mol Microbiol. 51: 395-405.

91. Minsky A, Shimoni E \& Frenkiel-Krispin D. 2002. Stress, order and survival. Nature Rev Mol Cell Biol. 3: 50-60.

92. Bohrmann B, Villigar W, Johanson R \& Kellenberger E. 1991. Coralline shape of the bacterial nucleoid after cryofixaction. J Bacteriol. 173: 3149-3158.

93. Case RB, Chang YP, Smith SB, Gore J, Cozzarelli NR \& Bustamante C. 2004. The bacterial condensin MukBEF compacts DNA into a repetitive, stable structure. Science. 305: 222-227.

94. Kimura K, Cuvier O \& Hirano T. 2001. Chromosome condensation by a human condensin complex in Xenopus egg extracts. $J$ Biol Chem. 276: $5417-5420$.

95. Kimura K, Hirono M, Kobayashi R \& Hirono T. 1998. Phosphorylation and activation of $13 \mathrm{~S}$ condensin by Cdc2 in vitro. Science. 282: 487-490.
96. Kimura K \& Hirono T. 1997. ATP-dependent positive supercoiling of DNA by $13 \mathrm{~S}$ condensin: A biochemical implication for chromosome condensation. Cell. 90: 625-634.

97. Lindow JC, Britton RA \& Grossman AD. 2002. Structural maintenance of chromosomes protein of Bacillus subtilis affects supercoiling in vivo. J Bacteriol 184: 5317-5322.

98. Niki H, Imamura R, Kitaoka M, Yamanaka K, Ogura T \& Hiraga S. 1992. E. coli MukB protein involved in chromosome partition forms a homodimer with a rod-and-hinge structure having DNA binding and ATP/GTP binding activities. EMBO J. 11: 5101-5109.

99. Hiraga S, Niki H, Ogura T, Ichinose C, Mori H, Ezaki B \& Jaffe A. 1989. Chromosomal partitioning in Escherichia coli: Novel mutants producing anucleate cells. J Bacteriol. 171: 1496-1505.

100. Hiraga S. 2000. Dynamic localization of bacterial and plasmid chromosomes. In Annual Review of Genetics (Campbell A, Anderson WW \& Jones EW eds), Vol 34, pp 21-59. Palo Alto, California.

101. Sawitzke JA \& Austin S. 2000. Suppression of chromosome segregation defects of Escherichia coli muk mutations in topoisomerase I. Proc Natl Acad Sci USA. 97: 1671-1676.

102. Weitao T, Nordström K \& Dasgupta S. 1999. Mutational suppression of $m u k B$ and seqA phenotypes might arise from their opposing influences on the Escherichia coli nucleoid structure. Mol Microbiol. 34: 157-168. 